\title{
CoLoRFul NNLO - Completely local subtractions for fully differential predictions at NNLO
}

\author{
Gábor Somogyi* \\ MTA-DE Particle Physics Research Group, Debrecen, Hungary \\ E-mail: gabor.somogyi@cern.ch
}

\section{Francesco Tramontano}

Dipartimento di Fisica, UniversitĹ di Napoli and INFN, Sezione di Napoli, I-80126 Naples, Italy

E-mail: francesco.tramontano@cern.ch

\section{Zoltán Trócsányi}

Institute of Physics, University of Debrecen, H-4010 Debrecen P.O.Box 105, Hungary

E-mail: zoltan.trocsanyidcern.ch

We present CoLoRFul NNLO, a completely local subtraction scheme for computing fully differential predictions at NNLO accuracy in QCD perturbation theory. As a first physical application and proof of concept we compute the fully differential decay rate of a Standard Model Higgs boson into b-quarks at NNLO.

12th International Symposium on Radiative Corrections (Radcor 2015) and LoopFest XIV (Radiative Corrections for the LHC and Future Colliders)

15-19 June 2015

UCLA Department of Physics \& Astronomy Los Angeles, CA, USA

\footnotetext{
* Speaker.
} 
When computing theoretical predictions for physical observables in quantum chromodynamics (QCD) beyond leading order in perturbation theory, one has to face among others the problem of infrared singularities. These singularities, which arise from integrations over unresolved momenta (momenta that are soft and/or collinear to one another), are known by the Kinoshita-Lee-Nauenberg theorem $[1,2]$ to cancel between real and virtual quantum corrections at any given order in perturbation theory for sufficiently inclusive (infrared-safe) observables. However, making this cancellation explicit in intermediate stages of the calculation is non-trivial already at next-to-leading order (NLO), where nevertheless several general solutions are known $[3,4,5,6,7,8]$. In the past decade or so, severe efforts have been made to devise such methods also at the next-to-next-toleading order (NNLO) in QCD perturbation theory and there are by now a number of approaches $[9,10,11,12,13,14]$ which have yielded physical predictions for one or several processes.

In particular in Ref. [15] we presented the first physical application of the CoLoRFul NNLO (Completely Local subtRactions for Fully differential predictions at NNLO) method [16, 17], which is a general subtraction scheme for computing QCD jet cross sections at NNLO accuracy. At present, the algorithm is worked out fully for processes with no colored particles in the initial state. Our intention was to devise a scheme which incorporates all the salient features of the most widely used NLO subtraction algorithms:

1. The subtraction scheme should be defined completely explicitly for arbitrary processes and all degrees of freedom (momentum, spin, color and flavor).

2. The algorithm should produce fully differential results in four dimensions, so that arbitrary detector cuts can be applied.

3. The subtraction terms should be fully local over the phase space of real emissions, including all color and spin correlations in infrared limits.

4. At least the poles of the integrated subtraction terms should be computed analytically, such that the correctness of the scheme can be rigorously proved.

5. It should be possible to restrict the subtractions to near the singular regions of the real emission phase space.

The general layout of the scheme is the following: consider the production of $m$ jets from a colorless initial state for simplicity. The cross section for this process in QCD perturbation theory is given by an expansion of the form $\sigma=\sigma^{\mathrm{LO}}+\sigma^{\mathrm{NLO}}+\sigma^{\mathrm{NNLO}}+\ldots$, where specifically the finite NNLO contribution is the sum of three terms

$$
\sigma^{\mathrm{NNLO}}=\int_{m+2} \mathrm{~d} \sigma_{m+2}^{\mathrm{RR}} J_{m+2}+\int_{m+1} \mathrm{~d} \sigma_{m+1}^{\mathrm{RV}} J_{m+1}+\int_{m} \mathrm{~d} \sigma_{m}^{\mathrm{VV}} J_{m},
$$

each separately infrared divergent in four dimensions. Indeed, $\mathrm{d} \sigma_{m+2}^{\mathrm{RR}}$ is singular over regions of the double real emission phase space where one or two partons become unresolved (soft and/or collinear); $\mathrm{d} \sigma_{m+1}^{\mathrm{RV}}$ is singular over regions of the real emission phase space where one parton becomes unresolved and furthermore contains explicit infrared $\varepsilon$-poles coming from one-loop matrix elements; finally $\mathrm{d} \sigma_{m}^{\mathrm{VV}}$ is not singular over the phase space if $J$ defines an infrared-safe observable 
but it contains explicit infrared $\varepsilon$-poles coming from one-loop squared and two-loop matrix elements. The basic idea of any subtraction scheme is to reshuffle the singularities between the three contributions in eq. (1) by subtracting and adding back suitably defined approximate cross sections, such that after this reshuffling, all three contributions become explicitly finite. In the CoLoRFul NNLO method, this rearrangement reads

$$
\begin{aligned}
& \mathrm{d} \sigma_{m+2}^{\mathrm{NNLO}}=\left\{\mathrm{d} \sigma_{m+2}^{\mathrm{RR}} J_{m+2}-\mathrm{d} \sigma_{m+2}^{\mathrm{RR}, \mathrm{A}_{2}} J_{m}-\left[\mathrm{d} \sigma_{m+2}^{\mathrm{RR}, \mathrm{A}_{1}} J_{m+1}-\mathrm{d} \sigma_{m+2}^{\mathrm{RR}, \mathrm{A}_{12}} J_{m}\right]\right\}_{\varepsilon=0}, \\
& \mathrm{~d} \sigma_{m+1}^{\mathrm{NNLO}}=\left\{\left[\mathrm{d} \sigma_{m+1}^{\mathrm{RV}}+\int_{1} \mathrm{~d} \sigma_{m+2}^{\mathrm{RR}, \mathrm{A}_{1}}\right] J_{m+1}-\left[\mathrm{d} \sigma_{m+1}^{\mathrm{RV}, \mathrm{A}_{1}}+\left(\int_{1} \mathrm{~d} \sigma_{m+2}^{\mathrm{RR}, \mathrm{A}_{1}}\right)^{\mathrm{A}_{1}}\right] J_{m}\right\}_{\varepsilon=0}, \\
& \mathrm{~d} \sigma_{m}^{\mathrm{NNLO}}=\left\{\mathrm{d} \sigma_{m}^{\mathrm{VV}}+\int_{2}\left[\mathrm{~d} \sigma_{m+2}^{\mathrm{RR}, \mathrm{A}_{2}}-\mathrm{d} \sigma_{m+2}^{\mathrm{RR}, \mathrm{A}_{12}}\right]+\int_{1}\left[\mathrm{~d} \sigma_{m+1}^{\mathrm{RV}, \mathrm{A}_{1}}+\left(\int_{1} \mathrm{~d} \sigma_{m+2}^{\mathrm{RR}, \mathrm{A}_{1}}\right)^{\mathrm{A}_{1}}\right]\right\}_{\varepsilon=0} J_{m} .
\end{aligned}
$$

Above, $\mathrm{d} \sigma_{m+2}^{\mathrm{RR}, \mathrm{A}_{2}}$ and $\mathrm{d} \sigma_{m+2}^{\mathrm{RR}, \mathrm{A}_{1}}$ regularize the double and single singular unresolved limits of $\mathrm{d} \sigma_{m+2}^{\mathrm{RR}}$, while $\mathrm{d} \sigma_{m+2}^{\mathrm{RR}, \mathrm{A}_{12}}$ serves a dual purpose: it regularizes $\mathrm{d} \sigma_{m+2}^{\mathrm{RR}, \mathrm{A}_{1}}$ in double unresolved limits and also $\mathrm{d} \sigma_{m+2}^{\mathrm{RR}, \mathrm{A}_{2}}$ in single unresolved ones. Furthermore, $\mathrm{d} \sigma_{m+1}^{\mathrm{RV}, \mathrm{A}_{1}}$ and $\left(\int_{1} \mathrm{~d} \sigma_{m+2}^{\mathrm{RR}, \mathrm{A}_{1}}\right)^{\mathrm{A}_{1}}$ cancel the kinematical singularities of $\mathrm{d} \sigma_{m+1}^{\mathrm{RV}}$ and $\int_{1} \mathrm{~d} \sigma_{m+2}^{\mathrm{RR}, \mathrm{A}_{1}}$ in single unresolved regions of phase space. Finally, provided the full construction is well-defined, the sum of integrated approximate cross sections in eq. (4) cancels the explicit $\varepsilon$-poles of $\mathrm{d} \sigma_{m}^{\mathrm{VV}}$, such that each of the three contributions is finite in four dimensions.

In order to define the approximate cross sections which appear in eqs. (2)-(4) explicitly, we use two key concepts that were at the core of general methods at NLO: (i) the infrared factorization of QCD squared matrix elements in unresolved limits and (ii) mappings of phase space which allow for integrating over unresolved momenta independently of the jet function. At NNLO, a new phenomena occurs that is not present at NLO: in double real radiation both single and double unresolved limits must be regularized. Hence, a third cornerstone of the construction, new at NNLO, is (iii) a process independent way of disentangling overlapping singularities both among the various double unresolved limits and also among the single and double unresolved ones.

The factorization properties of QCD squared matrix elements in all infrared limits relevant at NNLO have been known for quite some time both for double emission at tree level $[18,19]$ and for single emission from one-loop matrix elements [20, 21]. Nevertheless, the explicit implementation of the second and third points above pose a major challenge. We have presented a solution to the third point in Ref. [10] valid at NNLO, while in Ref. [22] we re-derived these results via an algorithm that generalizes to any order in perturbation theory, obtaining approximate matrix elements which have the correct limiting behavior in all infrared limits. However, those expressions still cannot be used as true subtraction terms, because they are only well-defined in strict soft and/or collinear limits. To construct true approximate cross sections, we must still specify the precise definition of subtraction terms away from the strict limits. This is accomplished by defining mappings from sets of $m+2$ and $m+1$ momenta to a set of $m$ momenta in such a way that the mappings not only lead to the exact factorization of phase space, but they also respect the delicate structure of cancellations among the various limit formulae in each infrared singular region. Such mappings, together with the explicit definition of approximate cross sections were presented in Ref. [16] for double real emission, and in Ref. [17] for real virtual emission. 
The subtraction terms in the CoLoRFul NNLO scheme are fully local, and it is possible to demonstrate the local cancellation of real emission singularities in any particular infrared limit. Figure 1 shows two examples for the case of double real emission. To produce the left panel, we have generated momentum configurations tending to a single soft limit and plotted the ratio of the sum of all subtractions to the double real emission matrix element for the production of a quarkantiquark pair and three gluons in electron-positron annihilation, relevant for a computation of $e^{+} e^{-} \rightarrow 3$ jets at NNLO. On the right panel the same procedure was applied but now the momentum configurations generated approached the limit where the momenta of the three gluons become collinear. Evidently as we close in on the limits the distributions of the ratio, centered on one, become ever narrower, demonstrating that the singularity is properly regularized in each case. We obtain similar results for real virtual emission, hence the $m+2$ and $m+1$ parton contributions in eqs. (2) and (3) are indeed finite and may be computed numerically by Monte Carlo techniques.
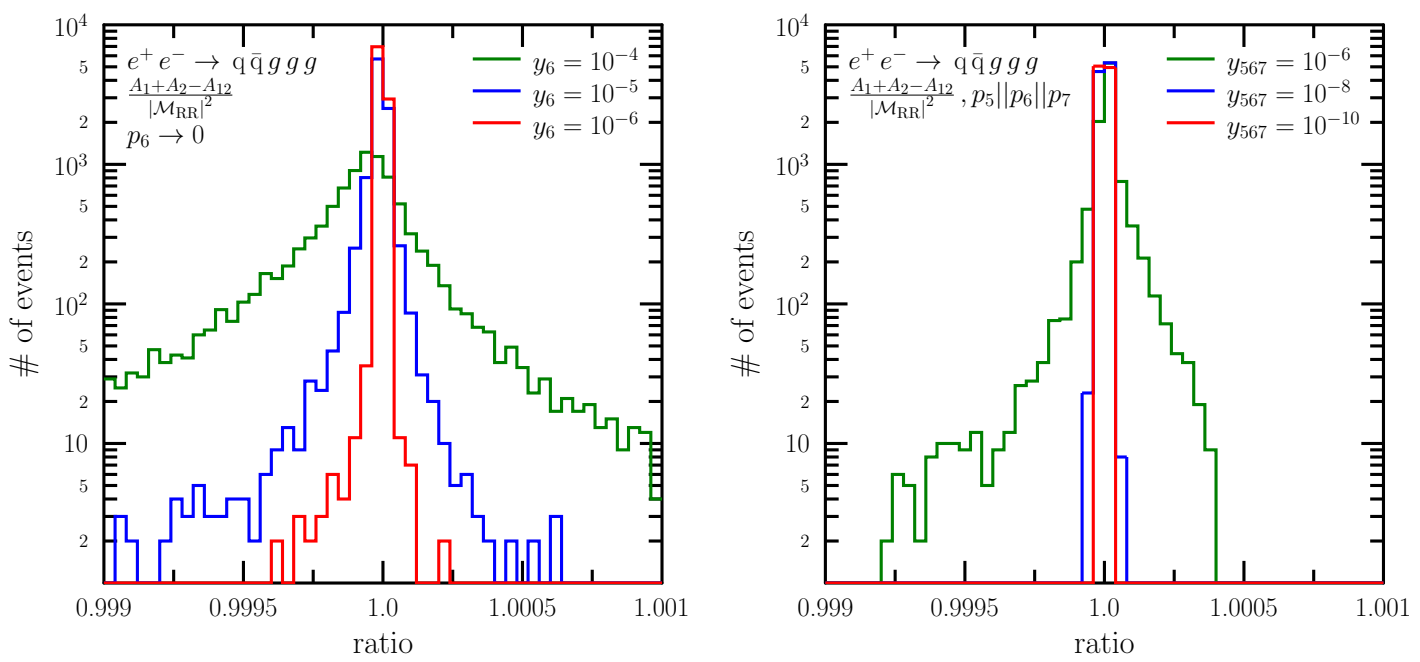

Figure 1: Ratio of the sum of all NNLO subtraction terms to the double real emission matrix element $e^{+} e^{-} \rightarrow q \bar{q} g g g$ in the single soft (left) and triple collinear (right) limits. The measure of softness is $y_{6}=$ $E_{6} / \sqrt{Q^{2}}$, while the measure of collinearity is $y_{567}=\left(p_{5}+p_{6}+p_{7}\right)^{2} / Q^{2}$.

Turning to the $m$ parton contribution in eq. (4), we note that the simultaneous factorization of phase space and matrix elements in all approximate cross sections allows us to integrate over the momenta of unresolved emissions independently of the process or observable being considered. We must also sum over the flavors of the unresolved partons. These steps together are denoted symbolically by $\int_{2}$ and $\int_{1}$ in eq. (4), the index indicating the number of unresolved partons. As a result of these manipulations, we obtain each integrated approximate cross section in the form of a product (in color space) of a lower point cross section times a singular insertion operator. Specifically, the sum of the four integrated approximate cross sections that appear in eq. (4) can be written as a singular operator times the Born cross section for producing $m$ partons, $\mathrm{d} \sigma_{m}^{\mathrm{B}}$, plus a different singular operator multiplying the one-loop virtual cross section for the production of $m$ partons, $\mathrm{d} \sigma_{m}^{\mathrm{V}}$. In a well-defined subtraction scheme all $\varepsilon$-poles of $\mathrm{d} \sigma_{m}^{\mathrm{VV}}$ must cancel against the 
poles of the integrated approximate cross sections, making the total $m$ parton contribution in eq. (4) finite in four dimensions.

The explicit computation of the various singular insertion operators is a laborious task that involves the evaluation of many cumbersome multi-dimensional phase space integrals. By way of example, we display one integral which arises during the integration of $\mathrm{d} \sigma_{m+2}^{\mathrm{RR}, \mathrm{A}_{2}}$ :

$$
\begin{aligned}
\mathscr{I}_{2 \mathscr{S}, 2} & \left(Y_{i k, Q} ; \varepsilon, y_{0}, d_{0}^{\prime}\right)=-\frac{4 \Gamma^{4}(1-\varepsilon)}{\pi \Gamma^{2}(1-\varepsilon)} \frac{B_{y_{0}}\left(-2 \varepsilon, d_{0}^{\prime}\right)}{\varepsilon} Y_{i k, Q} \int_{0}^{y_{0}} \mathrm{~d} y y^{-1-2 \varepsilon}(1-y)^{d_{0}^{\prime}-1+\varepsilon} \\
& \times \int_{-1}^{1} \mathrm{~d}(\cos \vartheta)(\sin \vartheta)^{-2 \varepsilon} \int_{-1}^{1} \mathrm{~d}(\cos \varphi)(\sin \varphi)^{-1-2 \varepsilon}[f(\vartheta, \varphi ; 0)]^{-1}\left[f\left(\vartheta, \varphi ; Y_{i k, Q}\right)\right]^{-1} \\
& \times\left[Y\left(y, \vartheta, \varphi ; Y_{i k, Q}\right)\right]^{-\varepsilon}{ }_{2} F_{1}\left(-\varepsilon,-\varepsilon, 1-\varepsilon, 1-Y\left(y, \vartheta, \varphi ; Y_{i k, Q}\right)\right),
\end{aligned}
$$

where

$$
f\left(\vartheta, \varphi ; Y_{i k, Q}\right)=1-2 \sqrt{Y_{i k, Q}\left(1-Y_{i k, Q}\right)} \sin \vartheta \cos \varphi-\left(1-2 Y_{i k, Q}\right) \chi \cos \vartheta,
$$

and

$$
Y(y, \vartheta, \varphi ; \chi)=\frac{4(1-y) Y_{i k, Q}}{[2(1-y)+y f(\vartheta, \varphi ; 0)]\left[2(1-y)+y f\left(\vartheta, \varphi ; Y_{i k, Q}\right)\right]}
$$

Evidently this integral is divergent in four dimensions and for an actual calculation, we must compute its Laurent expansions in $\varepsilon$, the parameter of dimensional regularization. To evaluate the integral in eq. (5), we employ the following strategy:

1. We begin by performing all integrations in terms of Mellin-Barnes integrals. The non-trivial angular integrals can be handled by the methods of Ref. [23].

2. The $\varepsilon$-pole structure of Mellin-Barnes integrals can be resolved by analytic continuation in an algorithmic way $[24,25]$. After analytic continuation, all pole coefficients are given in terms of finite Mellin-Barnes integrals.

3. We rewrite these finite Mellin-Barnes integrals as Euler-type integrals using the ideas of Ref. [26] to obtain a representation of the pole coefficients in terms of finite parametric integrals over a real domain.

4. These parametric integrals are finally evaluated in terms of multiple polylogarithms using modern techniques of symbolic integration.

5. The final result is simplified using the Hopf algebra structure of multiple polylogarithms [27]. After simplification the pole coefficients contain only ordinary polylogarithms. 
These steps lead to a complete analytic solution for the poles of eq. (5) (for $y_{0}=1$ and $d_{0}^{\prime}=3-3 \varepsilon$ ):

$$
\begin{aligned}
\mathscr{I}_{2 \mathscr{S}, 2}(Y ; \varepsilon, 1,3-3 \varepsilon)= \\
=\frac{1}{2 \varepsilon^{4}}-\frac{1}{\varepsilon^{3}}[\ln (Y)-3]+\frac{1}{\varepsilon^{2}}\left[2 \operatorname{Li}_{2}(1-Y)+\ln ^{2}(Y)-\pi^{2}-\left(\frac{2}{1-Y}\right.\right. \\
\left.\left.-\frac{1}{2(1-Y)^{2}}+\frac{9}{2}\right) \ln (Y)+\frac{1}{2(1-Y)}+16\right]+\frac{1}{\varepsilon}\left[\frac { 5 } { 3 } \left(\frac{18 \mathrm{Li}_{3}(1-Y)}{5}+\frac{6 \operatorname{Li}_{3}(Y)}{5}\right.\right. \\
\left.\quad-\frac{6 \operatorname{Li}_{2}(1-Y) \ln (Y)}{5}-\frac{2}{5} \ln ^{3}(Y)+\frac{3}{5} \ln (1-Y) \ln ^{2}(Y)+\pi^{2} \ln (Y)-\frac{78 \zeta_{3}}{5}\right) \\
+\left(\frac{3}{1-Y}-\frac{3}{4(1-Y)^{2}}+\frac{15}{4}\right)\left(2 \operatorname{Li}_{2}(1-Y)+\ln ^{2}(Y)\right)-6 \pi^{2}-\left(\frac{27}{2(1-Y)}\right. \\
\left.\left.\quad-\frac{13}{4(1-Y)^{2}}+\frac{91}{4}\right) \ln (Y)+\frac{19}{4(1-Y)}+\frac{163}{2}\right]+\mathrm{O}\left(\varepsilon^{0}\right) .
\end{aligned}
$$

Using the strategy outlined above, we have been able to compute the complete pole structure of the various singular insertion operators which appear in the integrated approximate cross sections. As discussed before, the final check of the correctness of our subtraction scheme is the demonstration that the explicit $\varepsilon$-poles of the double virtual cross section, $\mathrm{d} \sigma_{m}^{\mathrm{VV}}$, cancel against poles coming from the integrated approximate cross sections. We have established this cancellation analytically for $m=2$ and $m=3$ which is a very strong check of the computations.

As a first physical application and proof of concept of the CoLoRFul NNLO scheme, we computed the fully differential decay rate of a Standard Model Higgs boson into b-quarks at NNLO accuracy [15]. (This process was first calculated to NNLO accuracy in Ref. [28] using sector decomposition based on non-linear mappings.) Starting with the inclusive decay rate, our implementation of eqs. (2)-(4) for this process gives (for $\mu=m_{H}$ )

$$
\Gamma=\Gamma^{\mathrm{LO}}\left[1+\frac{\alpha_{\mathrm{s}}}{\pi} \frac{17}{3}+\left(\frac{\alpha_{\mathrm{s}}}{\pi}\right)^{2} 29.15(2)+\left(\alpha_{\mathrm{s}}^{3}\right)\right],
$$

in agreement with the known result $[29,30,31]$

$$
\Gamma=\Gamma^{\mathrm{LO}}\left[1+\frac{\alpha_{\mathrm{s}}}{\pi} \frac{17}{3}+\left(\frac{\alpha_{\mathrm{s}}}{\pi}\right)^{2} 29.146714 \ldots+\left(\alpha_{\mathrm{s}}^{3}\right)\right] .
$$

In Figure 2 we compute the inclusive decay rate at $\mu=m_{H} / 2$ and $\mu=2 m_{H}$ and compare it to the analytic result for the scale dependence, finding excellent agreement.

As our calculation is fully differential in four dimensions, we can also make predictions for any infrared-safe observable. To demonstrate the impact of NNLO corrections on differential distributions, we cluster the final state particles using the Durham jet algorithm [32] with resolution parameter $y_{\text {cut }}=0.05$ and order the resulting jets in energy. The top panel of Figure 3 shows the normalized energy distribution of the leading jet in the rest frame of the decaying Higgs boson for two-jet events. However, the energy of the most energetic jet is simply $E_{\max }=m_{H} / 2$ for two-parton kinematics, hence at leading order this distribution is just a delta function. Likewise, double unresolved subtractions for four parton final states, as well as single unresolved subtractions for three parton final states only contribute to the jet energy distribution at $E_{\max }=m_{H} / 2$. Thus, to demonstrate the method at work on an observable that has a non-trivial distribution already at 


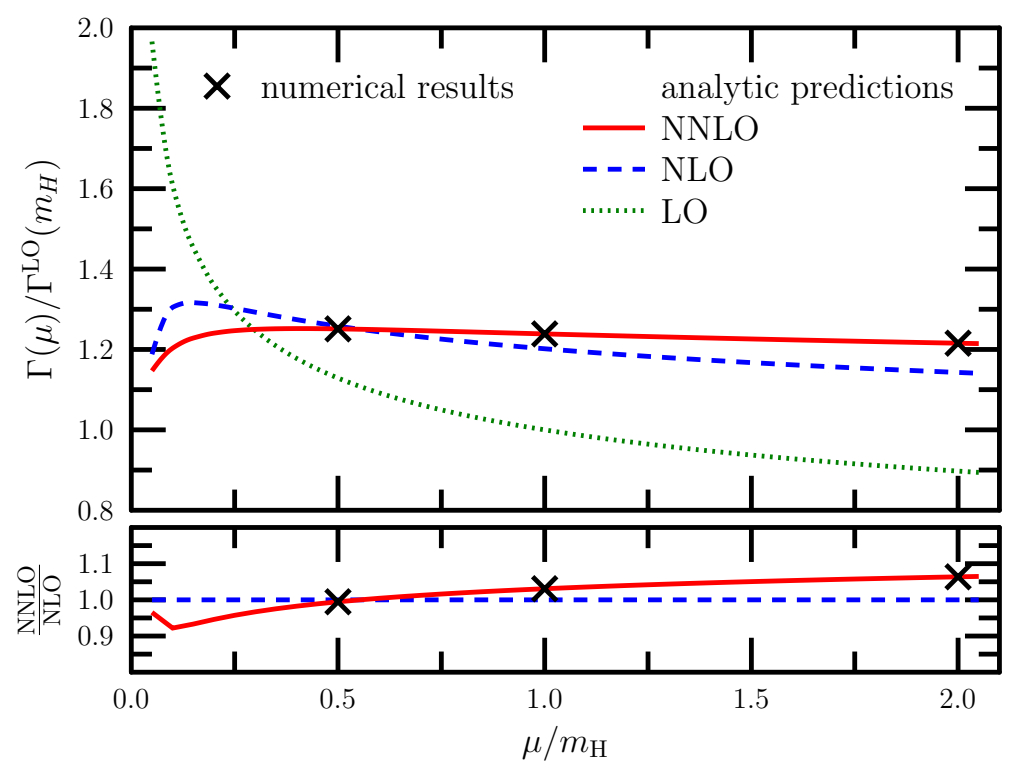

Figure 2: Scale dependence of the inclusive decay rate at LO, NLO and NNLO accuracy. The estimated uncertainty on the numerical results is too small to be appreciated.

leading order, we consider the absolute value of the pseudorapidity of the most energetic jet, $\left|\eta_{1}\right|$, with respect to an arbitrary axis. The bottom panel of Figure 3 shows the impact of higher order corrections on this distribution. The bands in Figure 3 represent renormalization scale dependence corresponding to the range $\mu \in\left[m_{H} / 2,2 m_{H}\right]$. In both examples, the uncertainty bands shrink when going from leading order to NNLO. For the pseudorapidity distribution, we furthermore observe the good convergence of the perturbative series, with the NNLO band falling within the NLO band. We note that the estimated numerical uncertainties form Monte Carlo integration are too small to be appreciated in Figure 3.

The CoLorFul NNLO subtraction scheme is constructed such that it is possible to constrain the subtractions to near the singular regions in phase space. The size of the constrained phase space is controlled by a dimensionless parameter $\alpha_{0} \in(0,1]$, where $\alpha_{0}=1$ corresponds to subtracting over the full real emission phase space. Physical predictions must of course be independent of $\alpha_{0}$ which is a highly non-trivial check on the correctness of the results. In Figure 4 we demonstrate the independence on $\alpha_{0}$ of the NNLO pseudorapidity distribution. In addition to offering a strong check, varying $\alpha_{0}$ also has implications for the numerical efficiency of the code. For example in the present case, setting $\alpha_{0}=0.1$ reduces the CPU time needed for the full calculation by about $60 \%$ as compared to the $\alpha_{0}=1$ case. This is due to the fact that with $\alpha_{0}<1$, not all subtraction terms need to be evaluated at each phase space point. In fact, for $\alpha_{0}=0.1$ we must compute on average only 14.5 subtraction terms out of a total 52 for $\alpha_{0}=1$. 
Durham clustering at $y_{\text {cut }}=0.05$

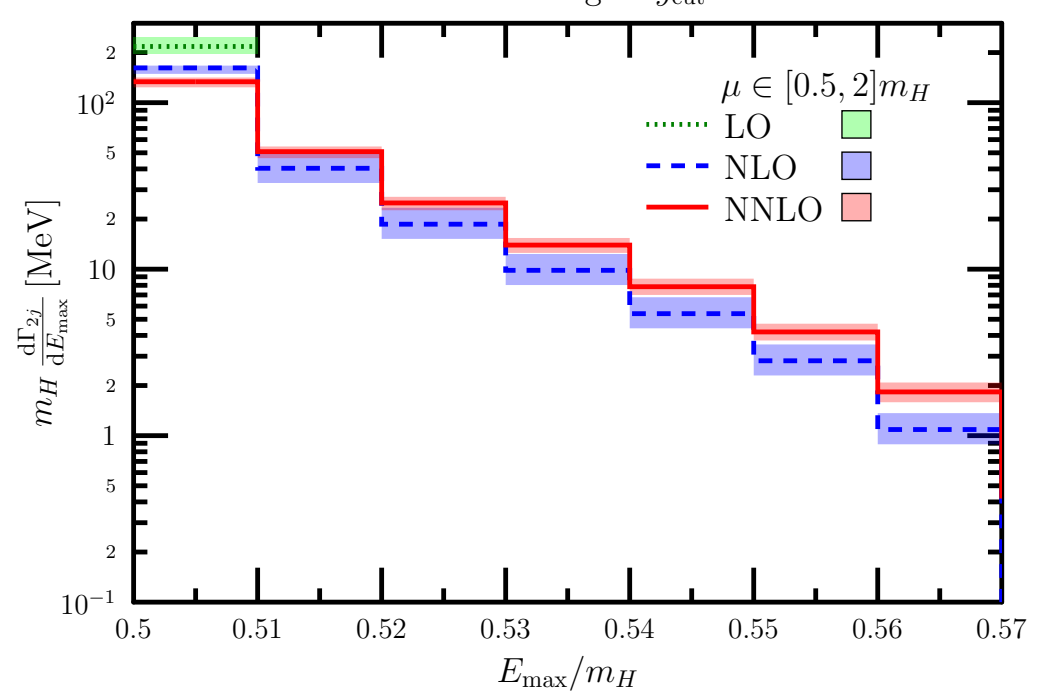

Durham clustering at $y_{\text {cut }}=0.05$

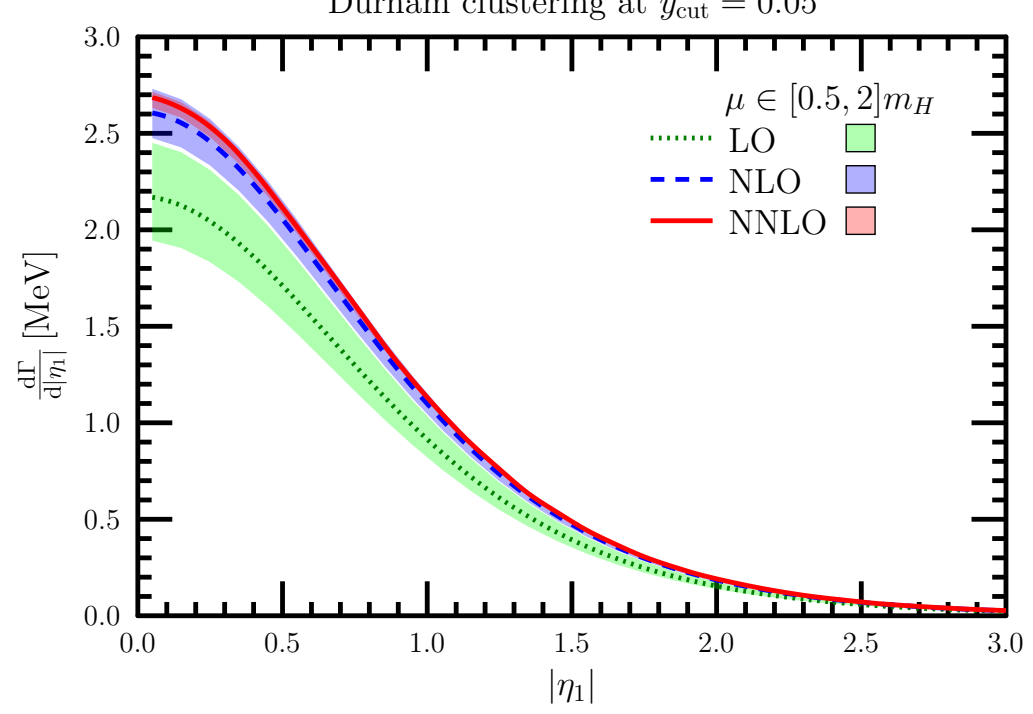

Figure 3: The normalized distribution of the leading jet energy $E_{\max }$ (top) and the distribution of the absolute value of the pseudorapidity $\left|\eta_{1}\right|$ of the most energetic jet (bottom) at LO, NLO and NNLO accuracy. The bands show the dependence on the renormalization scale corresponding to the range $\mu \in\left[m_{H} / 2,2 m_{H}\right]$. Jets were clustered using the Durham algorithm with resolution parameter $y_{\text {cut }}=0.05$. 


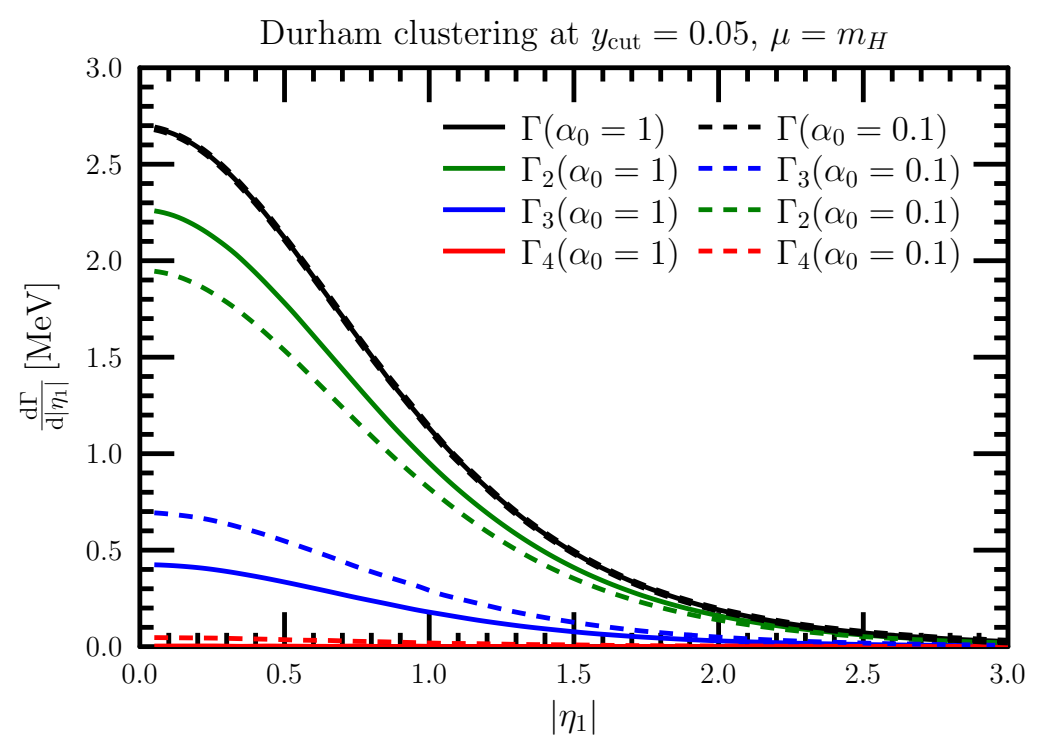

Figure 4: The distribution of the absolute value of the pseudorapidity $\left|\eta_{1}\right|$ of the most energetic jet at NNLO accuracy. The green, blue and red curves represent the contributions of the two-, three- and four-parton final states to the full result (black). Solid lines are obtained with unconstrained subtraction $\left(\alpha_{0}=1\right)$, while dashed lines correspond to $\alpha_{0}=0.1$.

We presented the CoLoRFul NNLO (Completely Local subtRactions for Fully differential predictions at NNLO) framework for computing QCD jet cross sections at NNLO accuracy. CoLoRFul NNLO is a completely local and fully differential subtraction scheme valid for arbitrary processes at NNLO. The construction of the subtraction terms is based on the known infrared factorization formulae for QCD squared matrix elements. Computing the integrated approximate cross sections is feasible with modern integration techniques, hence it is possible to verify the correctness of the scheme analytically. As a first physical application and proof of concept we presented a computation of the fully differential decay of a Standard Model Higgs boson into b-quarks at NNLO accuracy. Presently CoLoRFul NNLO is worked out in full detail for processes with no colored particles in the initial state, however the extension to hadron-induced processes is conceptually understood and is work in progress.

\section{References}

[1] T. Kinoshita, J. Math. Phys. 3, 650 (1962).

[2] T. D. Lee and M. Nauenberg, Phys. Rev. 133, B1549 (1964).

[3] W. T. Giele and E. W. N. Glover, Phys. Rev. D 46, 1980 (1992).

[4] W. T. Giele, E. W. N. Glover and D. A. Kosower, Nucl. Phys. B 403, 633 (1993) [hep-ph/9302225].

[5] S. Frixione, Z. Kunszt and A. Signer, Nucl. Phys. B 467, 399 (1996) [hep-ph/9512328]. 
[6] S. Catani and M. H. Seymour, Nucl. Phys. B 485, 291 (1997) [Nucl. Phys. B 510, 503 (1998)] [hep-ph/9605323].

[7] Z. Nagy and Z. Trócsányi, Nucl. Phys. B 486, 189 (1997) [hep-ph/9610498].

[8] S. Frixione, Nucl. Phys. B 507, 295 (1997) [hep-ph/9706545].

[9] C. Anastasiou, K. Melnikov and F. Petriello, Phys. Rev. D 69, 076010 (2004) [hep-ph/0311311].

[10] G. Somogyi, Z. Trócsányi and V. Del Duca, JHEP 0506, 024 (2005) [hep-ph/0502226].

[11] A. Gehrmann-De Ridder, T. Gehrmann and E. W. N. Glover, JHEP 0509, 056 (2005) [hep-ph/0505111].

[12] S. Catani and M. Grazzini, Phys. Rev. Lett. 98, 222002 (2007) [hep-ph/0703012].

[13] M. Czakon, Phys. Lett. B 693, 259 (2010) [arXiv:1005.0274 [hep-ph]].

[14] M. Czakon and D. Heymes, Nucl. Phys. B 890, 152 (2014) [arXiv:1408.2500 [hep-ph]].

[15] V. Del Duca, C. Duhr, G. Somogyi, F. Tramontano and Z. Trócsányi, JHEP 1504, 036 (2015) [arXiv:1501.07226 [hep-ph]].

[16] G. Somogyi, Z. Trócsányi and V. Del Duca, JHEP 0701, 070 (2007) [hep-ph/0609042].

[17] G. Somogyi and Z. Trócsányi, JHEP 0701, 052 (2007) [hep-ph/0609043].

[18] J. M. Campbell and E. W. N. Glover, Nucl. Phys. B 527, 264 (1998) [hep-ph/9710255].

[19] S. Catani and M. Grazzini, Nucl. Phys. B 570, 287 (2000) [hep-ph/9908523].

[20] Z. Bern, V. Del Duca, W. B. Kilgore and C. R. Schmidt, Phys. Rev. D 60, 116001 (1999) [hep-ph/9903516].

[21] S. Catani and M. Grazzini, Nucl. Phys. B 591, 435 (2000) [hep-ph/0007142].

[22] Z. Nagy, G. Somogyi and Z. Trócsányi, hep-ph/0702273 [HEP-PH].

[23] G. Somogyi, J. Math. Phys. 52, 083501 (2011) [arXiv:1101.3557 [hep-ph]].

[24] M. Czakon, Comput. Phys. Commun. 175, 559 (2006) [hep-ph/0511200].

[25] A. V. Smirnov and V. A. Smirnov, Eur. Phys. J. C 62, 445 (2009) [arXiv:0901.0386 [hep-ph]].

[26] C. Anastasiou, C. Duhr, F. Dulat and B. Mistlberger, JHEP 1307, 003 (2013) [arXiv:1302.4379 [hep-ph]].

[27] C. Duhr, JHEP 1208, 043 (2012) [arXiv:1203.0454 [hep-ph]].

[28] C. Anastasiou, F. Herzog and A. Lazopoulos, JHEP 1203, 035 (2012) [arXiv:1110.2368 [hep-ph]].

[29] S. G. Gorishnii, A. L. Kataev, S. A. Larin and L. R. Surguladze, Mod. Phys. Lett. A 5, 2703 (1990).

[30] S. G. Gorishnii, A. L. Kataev, S. A. Larin and L. R. Surguladze, Phys. Rev. D 43, 1633 (1991).

[31] P. A. Baikov, K. G. Chetyrkin and J. H. Kuhn, Phys. Rev. Lett. 96, 012003 (2006) [hep-ph/0511063].

[32] S. Catani, L. Trentadue, G. Turnock and B. R. Webber, Nucl. Phys. B 407, 3 (1993). 